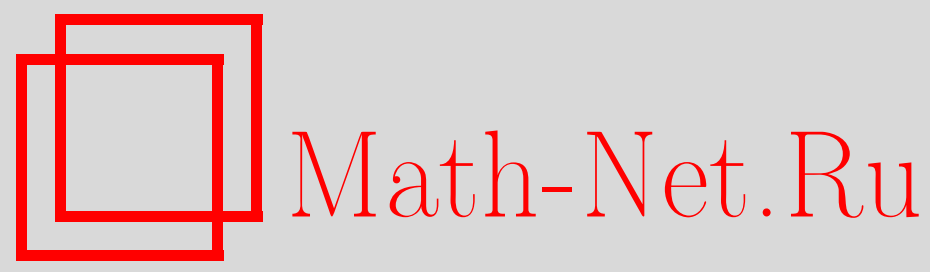

Е. И. Тимошенко, Центр некоторых разрешимых групп с одним определяющим соотношением, Матем. заметки, 1998, том 64, выпуск 6, 925-931

DOI: https://doi.org/10.4213/mzm1471

Использование Общероссийского математического портала Math-Net.Ru подразумевает, что вы прочитали и согласны с пользовательским соглашением http://www.mathnet.ru/rus/agreement

Параметры загрузки:

IP : 3.85 .7 .115

26 апреля 2023 г., 16:34:56

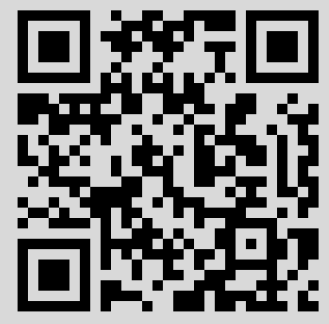




\section{ЦЕНТР НЕКОТОРЫХ РАЗРЕШИМЫХ ГРУПП С ОДНИМ ОПРЕДЕЛЯЮЩИМ СООТНОШЕНИЕМ}

\section{Е.И. Тимошенко}

Найдены необходимые и достаточные условия, при которых центр метабелевых групп с одним определяющим соотношением нетривиален. Описан центр таких групп. Исследован центр групп вида $F / N^{\prime}\langle g\rangle^{F}$ при некоторых ограничениях. С помощью другой техники усилен ранее полученный результат А. $\Phi$. Красникова и автора о центре групп указанного вида.

Библиограффия: 8 названий.

В Коуровской тетради [1] М. И. Каргаполов в 1973 году поставил следующий вопрос.

Вопрос [1, вопрос 4.33a)]. Пусть $\mathfrak{R}_{n}-$ класс групn с одним определяюшим соотношением в многообразии п-ступенно разрешимых групп. При каких условиях $\mathfrak{R}_{n}$-группа обладает нетривиальным иентром? Может ли быть нетривиальным

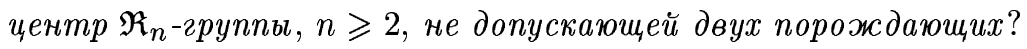

В работе автора [2] было доказано, что $\mathfrak{R}_{2}$-группа имеет тривиальный центр, если она не допускает двух порождающих элементов.

В книге избранных трудов М.И. Каргаполова [3] собраны его вопросы по теории групп и приведены комментарии к ним В.Н. Ремесленникова, отметившего, что ответ на первую часть вопроса не получен.

В данной заметке приведены необходимые и достаточные условия того, что $\mathfrak{R}_{2}$-групша имеет нетривиальный центр, и дано описание центра.

В совместной работе [4] А.Ф. Красникова и автора исследовался центр групп вида $F / N^{\prime}\langle g\rangle^{F}$ при некоторых ограничениях. В частности, предполагалось, что группа $F / N$ упорядочиваема. Использование кольца косых многочленов Лорана дает возможность доказать эту теорему в более общей формулировке.

1. Пусть $\mathfrak{A}$-многообразие всех абелевых груп, а $[x, y]=x^{-1} y^{-1} x y$. Можно указать очевидные случаи, когда центр $\mathfrak{R}_{n}$-группы нетривиален. Например, если $G=\left\langle x_{1}, x_{2}\right.$ : $\left.g=1, \mathfrak{A}^{n}\right\rangle$ и $g=\left[x_{1}, x_{2}\right]$ или $g$ - примитивньй элемент в группе $F_{2}\left(\mathfrak{A}^{n}\right)$. Можно найти и другие примеры $\mathfrak{R}_{n}$-групп, имеющих нетривиальный центр. Если ограничиться случаем $n=2$, то, как следует из [2], нужно рассматривать лишь групшы с двумя порождающими. Покажем, например, что центр групшы $G_{1}=\left\langle x_{1}, x_{2}: x_{1}^{2}\left[x_{1}, x_{2}\right]=1, \mathfrak{A}^{2}\right\rangle$ нетривиален, а именно, элемент $z=x_{2}^{2}$ является центральным в группе $G_{1}$.

Работа выполнена при поддержке Российского фонда фундаментальных исследований, грант № 96-01-01948. 
Пусть $R$ - нормальное замькание элемента $g=x_{1}^{2}\left[x_{1}, x_{2}\right]$ в свободной метабелевой группе $S=F_{2}\left(\mathfrak{A}^{2}\right)$. Достаточно доказать, что $\left[x_{1}, z\right]$ - элемент из $R$.

Имеем

$$
\left[g, x_{1}\right]=\left[x_{1}, x_{2}\right]^{\bar{x}_{1}-1}, \quad\left[g, x_{2}\right]=\left[x_{1}, x_{2}\right]^{\bar{x}_{1}+\bar{x}_{2}}, \quad\left[x_{1}, x_{2}^{2}\right]=\left[x_{1}, x_{2}\right]^{1+\bar{x}_{1}}
$$

где черта означает образ элемента из $S$ в группе $\bar{S}=S / S^{\prime}$. Так как $1+\bar{x}_{2}=(-1)\left(\bar{x}_{1}-1\right)+$ $\left(\bar{x}_{1}+\bar{x}_{2}\right)$, то $\left[x_{1}, z\right]=\left[g, x_{1}\right]^{-1}\left[g, x_{2}\right]$, т.е. $\left[x_{1}, z\right] \in R$.

Вместе с тем можно доказать, что групшы $G_{m}=\left\langle x_{1}, x_{2}: x_{1}^{m}=1, \mathfrak{A}^{2}\right\rangle$ имеют тривиальньй центр при $m \geqslant 2$.

Следующая теорема дает необходимые и достаточные условия того, что центр $\mathfrak{R}_{2}$-группы является нетривиальньм.

Теорема 1. Пусть $S$ - свободная метабелева группа с базисом $x_{1}, x_{2}, G=\left\langle x_{1}\right.$, $\left.x_{2}: g=1, \mathfrak{A}^{2}\right\rangle$ - метабелева группа с двумя порожсдаюшими и одним определяющим соотношением, $\bar{S}=S / S^{\prime}, g=x_{1}^{n}\left[x_{1}, x_{2}\right]^{\alpha}, n \geqslant 0, \alpha \in \mathbb{Z}(\bar{S}), \alpha=\sum_{i=1}^{l} n_{i} \bar{g}_{i}$, әде $n_{i} \in \mathbb{Z}, \bar{g}_{i} \in \bar{S}$. Пусть $H-$ подгруппа $\bar{S}$, порожденная әлементами $\bar{g}_{i}^{-1} \bar{g}_{j}$, когда $i, j$ пробегают все, необязательно разные, значения от 1 до l. Тогда

1) чентр группы $G$ есть свободная абелева группа ранга 2 , если $n=0$ и $\alpha-$ единииа кольиа $\mathbb{Z}(\bar{S})$

2) чентр группь $G$ есть бесконечная ииклическая группа, если $n=0, H$ - бесконечная ииклическая группа и элемент $\alpha$ является делителем әлемента $\left(\bar{h}^{m}-1\right) /(\bar{h}-1)$ для некоторого челого $m>0$ и некоторого примитивного әлемента $h$ из $S$;

3) иентр группь $G$ есть бесконечная ииклическая группа, если $n>0$ идля некоторого иелого $l \neq 0$ и некоторых элементов $A, B$ из фундаментального идеала $\Delta$ кольиа $\mathbb{Z}(\bar{S})$ имеет место

$$
\bar{x}_{2}^{n l}-1=A \alpha\left(1-\bar{x}_{1}\right)+B\left(\alpha\left(1-\bar{x}_{2}\right)+\frac{\bar{x}_{1}^{n}-1}{\bar{x}_{1}-1}\right)
$$

4) в остальных случаях иентр $\mathbb{Z}(G)$ группь $G$ тривиален;

5) иентр әруппы $G$ не содержит нетривиальных әлементов из коммутан$m a G^{\prime}$.

ДокАЗАТЕЛЬСТво разобьем на несколько случаев в зависимости от расположения элемента $g$ в групе $S$.

Случай 1. $g \in S^{\prime}, g=\left[x_{1}, x_{2}\right]^{\alpha}$.

Покажем, что в этом случае $Z(G) \cap G^{\prime}=1$.

Предположим, что $z=\left[x_{1}, x_{2}\right]^{\beta}$, где $\beta \in \mathbb{Z}(\bar{S})$,- центральньй элемент в группе $G$. Тогда для некоторых $\beta_{1}, \beta_{2}$ из $\mathbb{Z}(\bar{S})$ имеем

$$
\left[z, x_{1}\right]=\left[x_{1}, x_{2}\right]^{\beta\left(\bar{x}_{1}-1\right)}=\left[x_{1}, x_{2}\right]^{\alpha \beta_{1}}, \quad\left[z, x_{2}\right]=\left[x_{1}, x_{2}\right]^{\beta\left(\bar{x}_{2}-1\right)}=\left[x_{1}, x_{2}\right]^{\alpha \beta_{2}}
$$

Значит,

$$
\beta\left(\bar{x}_{1}-1\right)=\alpha \beta_{1}, \quad \beta\left(\bar{x}_{2}-1\right)=\alpha \beta_{2}
$$


Если $\beta_{1}$ делится на $\bar{x}_{1}-1$ или $\beta_{2}$ делится на $\bar{x}_{2}-1$ в $\mathbb{Z}(\bar{S})$, то $z=1$ в группе $G$. Предположим, что это не так, т.е. $\alpha=\alpha^{\prime}\left(\bar{x}_{1}-1\right)\left(\bar{x}_{2}-1\right)$. Тогда из (1) получаем

$$
\beta=\beta^{\prime}\left(\bar{x}_{1}-1\right)\left(\bar{x}_{2}-1\right) .
$$

Следовательно,

$$
\beta^{\prime}\left(\bar{x}_{1}-1\right)=\alpha^{\prime} \beta_{1}, \quad \beta^{\prime}\left(\bar{x}_{2}-1\right)=\alpha^{\prime} \beta_{2} .
$$

Так как процесс деления $\alpha$ и $\beta$ на $\left(\bar{x}_{1}-1\right)\left(\bar{x}_{2}-1\right)$ должен оборваться, то $z$ - элемент из $R=\langle g\rangle^{S}$.

Пусть центральный элемент $z$ лежит в $S \backslash S^{\prime}$.

Выберем базис $y_{1}, y_{2}$ группы $S$ так, что $z=y_{1}^{m}\left[y_{1}, y_{2}\right]^{\beta}$, где $m>0, \beta \in \mathbb{Z}(\bar{S})$.

Убедимся сначала, что если $z=y_{1}^{m}\left[y_{1}, y_{2}\right]^{\beta}$ - центральный элемент в групе $G$, то $z=y_{1}^{m}$ в группе $G$. Действительно,

$$
\left[z, y_{1}\right]=\left[y_{1}, y_{2}\right]^{\beta\left(\bar{y}_{1}-1\right)}, \quad\left[z, y_{2}\right]=\left[y_{1}, y_{2}\right]^{\left(\bar{y}_{1}^{m}-1\right) /\left(\bar{y}_{1}-1\right)+\beta\left(\bar{y}_{2}-1\right)} .
$$

Запишем элемент $g$ в виде $g=\left[y_{1}, y_{2}\right]^{\gamma}$, где $\gamma$ - некоторый элемент из $\mathbb{Z}(\bar{S})$. Так как $\left[z, y_{1}\right]$ и $\left[z, y_{2}\right]$ - элементы из $R$, найдутся $\gamma_{1}, \gamma_{2} \in \mathbb{Z}(\bar{S})$ такие, что

$$
\begin{gathered}
\beta\left(\bar{y}_{1}-1\right)=\gamma \gamma_{1}, \\
\frac{\bar{y}_{1}^{m}-1}{\bar{y}_{1}-1}+\beta\left(\bar{y}_{2}-1\right)=\gamma \gamma_{2} .
\end{gathered}
$$

Применим к (3) отображение $\varepsilon: \mathbb{Z}(\bar{S}) \rightarrow \mathbb{Z}$. Так как $\varepsilon\left(\left(\bar{y}_{1}^{m}-1\right) /\left(\bar{y}_{1}-1\right)\right)=m \neq 0$, то $\varepsilon(\gamma) \neq 0$. Значит, элемент $\gamma$ не делится на $\bar{y}_{1}-1$. Поэтому из $(2)$ получим $\beta=\gamma \gamma_{1}^{\prime}$ для некоторого $\gamma_{1}^{\prime} \in \mathbb{Z}(\bar{S})$. Значит,

$$
z g^{-\gamma_{1}^{\prime}}=y_{1}^{m}\left[y_{1}, y_{2}\right]^{\beta-\gamma \gamma_{1}^{\prime}}=y_{1}^{m} .
$$

Таким образом, можно считать, что $\beta=0$. Тогда из $(3)$ заключаем, что $\left(\bar{y}_{1}^{m}-1\right) /\left(\bar{y}_{1}-1\right)$ делится на $\gamma$.

Определим связь между элементами $\alpha$ и $\gamma$. Известно [5], что любой автоморфизм группы $S$ индуцирован некоторым автоморфизмом свободной группы $F_{2}$ с базисом $u_{1}, u_{2}$. Любой автоморфизм группы $F_{2}$ переводит коммутатор $\left[u_{1}, u_{2}\right]$ в элемент $\left[u_{1}, u_{2}\right]^{ \pm v}$, где $v \in F_{2}$ (см., например, [6]). Поэтому любой автоморфизм группы $S$ переводит коммутатор $\left[x_{1}, x_{2}\right]$ в элемент $\left[x_{1}, x_{2}\right]^{ \pm w}$, где $w \in S$.

Рассмотрим автоморфизм $\varphi$ группы $S: \varphi=\left\{x_{1} \mapsto y_{1}, x_{2} \mapsto y_{2}\right\}$. Тогда

$$
\left[y_{1}, y_{2}\right]=\left[\varphi\left(x_{1}\right), \varphi\left(x_{2}\right)\right]=\left[x_{1}, x_{2}\right]^{ \pm w}
$$

для некоторого $w \in S$. Поэтому

$$
g=\left[x_{1}, x_{2}\right]^{\alpha}=\left[y_{1}, y_{2}\right]^{\gamma}=\left[x_{1}, x_{2}\right]^{ \pm w \gamma},
$$

т.е. $\alpha= \pm w \gamma$. Значит, элемент $\left(\bar{y}_{1}^{m}-1\right) /\left(\bar{y}_{1}-1\right)$ делится на $\alpha$.

Обратно, если для некоторого примитивного элемента $y_{1}$ из $S$ и некоторого $m>0$ элемент $\left(\bar{y}_{1}^{m}-1\right) /\left(\bar{y}_{1}-1\right)$ делится на $\alpha$ в кольце $\mathbb{Z}(\bar{S})$, то элемент $y_{1}^{m}$ является центральным, поскольку условия (2) и (3) для него вьполнены. 
Проверим, что $\mathbb{Z}(G)$ - бесконечная циклическая группа, если вьполнены условия утверждения 2) теоремы. Действительно, в этом случае $l>1$, так как $H$ - бесконечная циклическая группа. Поэтому примитивный элемент $y_{1}$ определен однозначно по модулю $S^{\prime}$ элементом $\alpha$. Из того, что $z=y_{1}^{m} c, c \in S^{\prime},-$ центральный элемент в группе $G$, следует $z=y_{1}^{m}$ в $G$. Значит, $\mathbb{Z}(G)$ - бесконечная циклическая групша, порожденная степенью элемента $y_{1}$.

Случай 2. $g \in S \backslash S^{\prime}, g=x_{1}^{n}\left[x_{1}, x_{2}\right]^{\alpha}, n>0$.

Обоснование того, что $Z(G) \cap G^{\prime}=1$, является практически дословным повторением п. 2 доказательства теоремы из [2].

Найдем необходимые и достаточные условия того, чтобы элемент $z \in S \backslash S^{\prime}$ был центральным.

Пусть $z=x_{1}^{m_{1}} x_{2}^{m_{2}}\left[x_{1}, x_{2}\right]^{\beta^{\prime}}$, где $m_{1}, m_{2} \in \mathbb{Z}, \beta^{\prime} \in \mathbb{Z}(\bar{S})$, является центральным элементом в групше $G$.

Рассмотрим свободную двуступенную нильпотентную группу $F_{2}\left(\mathbb{N}_{2}\right)$ ранга 2 с базисом $a_{1}, a_{2}$ и гомоморфизм $\theta=\left\{x_{1} \mapsto a_{1}, x_{2} \mapsto a_{2}\right\}$. Так как $z$ - центральный элемент, то $[z, S] \leqslant[\langle g\rangle, S]$, где $\langle g\rangle$ - групша, порожденная элементом $g$.

Применим $\theta$ к этому включению. Тогда $\left[a_{1}, a_{2}\right]^{m_{1}},\left[a_{1}, a_{2}\right]^{m_{2}}$ - элементы из группы $\left\langle\left[a_{1}, a_{2}\right]^{n}\right\rangle$. Поэтому $m_{1}=n l_{1}, m_{2}=n l$. Значит, в групше $G$ элемент $z$ равен элементу $x_{2}^{n l}\left[x_{1}, x_{2}\right]^{\beta}$, причем $l \neq 0$, так как $z \neq 1$ в $G$.

Итак, пусть $z=x_{2}^{n l}\left[x_{1}, x_{2}\right]^{\beta}$ - неединичньй центральньй элемент в группе $G$. Тогда

$$
\left[x_{1}, z\right]=\left[x_{1}, x_{2}\right]^{\left(\bar{x}_{2}^{n l}-1\right) /\left(\bar{x}_{2}-1\right)+\beta\left(1-\bar{x}_{1}\right)}, \quad\left[x_{2}, z\right]=\left[x_{1}, x_{2}\right]^{\beta\left(1-\bar{x}_{2}\right)}
$$

- элементы из $R$. Так как $R=\langle g\rangle[\langle g\rangle, S]$, то элементы $\left[x_{1}, z\right]$ и $\left[x_{2}, z\right]$ лежат в подгруппе $[\langle g\rangle, S]$. Нормальная подгрупа $[\langle g\rangle, S]$ порождена, как $\mathbb{Z}(\bar{S})$-модуль, элементами

$$
\left[x_{1}^{n}\left[x_{1}, x_{2}\right]^{\alpha}, x_{1}\right]=\left[x_{1}, x_{2}\right]^{\alpha\left(\bar{x}_{1}-1\right)}, \quad\left[x_{1}^{n}\left[x_{1}, x_{2}\right]^{\alpha}, x_{2}\right]=\left[x_{1}, x_{2}\right]^{\left(\bar{x}_{1}^{n}-1\right) /\left(\bar{x}_{1}-1\right)+\alpha\left(\bar{x}_{2}-1\right)} .
$$

Поэтому элемент $z$ является центральньм в группе $G$ тогда и только тогда, когда найдутся $\gamma_{1}, \gamma_{2}, \gamma_{3}, \gamma_{4} \in \mathbb{Z}(\bar{S})$ такие, что

$$
\begin{gathered}
\frac{\bar{x}_{2}^{n l}-1}{\bar{x}_{2}-1}+\beta\left(1-\bar{x}_{1}\right)=\gamma_{1} \alpha\left(\bar{x}_{1}-1\right)+\gamma_{2}\left(\frac{\bar{x}_{1}^{n}-1}{\bar{x}_{1}-1}+\alpha\left(\bar{x}_{2}-1\right)\right), \\
\beta\left(1-\bar{x}_{2}\right)=\gamma_{3} \alpha\left(\bar{x}_{1}-1\right)+\gamma_{4}\left(\frac{\bar{x}_{1}^{n}-1}{\bar{x}_{1}-1}+\alpha\left(\bar{x}_{2}-1\right)\right) .
\end{gathered}
$$

Система уравнений (4) эквивалентна одному уравнению

$\bar{x}_{2}^{n l}-1=\left(\gamma_{1}\left(\bar{x}_{2}-1\right)+\gamma_{3}\left(1-\bar{x}_{1}\right)\right) \alpha\left(\bar{x}_{1}-1\right)+\left(\gamma_{2}\left(\bar{x}_{2}-1\right)+\gamma_{4}\left(1-\bar{x}_{1}\right)\right)\left(\frac{\bar{x}_{1}^{n}-1}{\bar{x}_{1}-1}+\alpha\left(\bar{x}_{2}-1\right)\right)$.

Таким образом, если группа $G$ имеет нетривиальньй центр, то найдется $l \neq 0$ и элементы $A, B$ из $\Delta$ такие, что

$$
x_{2}^{n l}-1=A \alpha\left(\bar{x}_{1}-1\right)+B\left(\frac{\bar{x}_{1}^{n}-1}{\bar{x}_{1}-1}+\alpha\left(\bar{x}_{2}-1\right)\right) .
$$

Верно и обратное, т.е. если существует $l \neq 0$ и $A, B \in \Delta$ удовлетворяют уравнению (5), то группа $G$ имеет нетривиальньй центр. 
Покажем, что центр группы $G$ есть бесконечная циклическая группа, если условия утверждения 3) теоремы выполнены.

Действительно, все центральные элементы в группе $G$ имеют вид $x_{2}^{n l}\left[x_{1}, x_{2}\right]^{\beta}$. Пусть $z_{1}=x_{2}^{n l_{1}}\left[x_{1}, x_{2}\right]^{\beta_{1}}$ и $z_{2}=x_{2}^{n l_{2}}\left[x_{1}, x_{2}\right]^{\beta_{2}}$ - два центральных неединичных элемента из $G$ и $d$ - наибольший общий делитель чисел $l_{1}, l_{2}$. Тогда $z=x_{2}^{n d}\left[x_{1}, x_{2}\right]^{\beta_{3}}$ - также центральньй элемент в $G$ при некотором $\beta_{3} \in \mathbb{Z}(\bar{S})$. Но тогда найдется $t \in \mathbb{Z}$ такое, что

$$
x_{2}^{n l_{1}}\left[x_{1}, x_{2}\right]^{\beta_{1}} z^{-t}=\left[x_{1}, x_{2}\right]^{\delta}
$$

- центральный элемент. Так как $Z(G) \cap G^{\prime}=1$, то $z_{1}=z^{t}$. Теорема доказана.

Рассмотрим несколько примеров.

ПРИМЕР 1. $n=2, \alpha=1$.

При этих значениях уравнение (5) имеет решение $A=1-\bar{x}_{2}, B=\bar{x}_{2}-1, l=1$. Этим значениям соответствует группа $G_{1}=\left\langle x_{1}, x_{2}: x_{1}^{2}\left[x_{1}, x_{2}\right]=1, \mathfrak{A}^{2}\right\rangle$, имеющая нетривиальный центр.

ПРИМЕР 2. $n \geqslant 2, \alpha=0$.

Из (5) получаем

$$
\frac{\bar{x}_{2}^{n l}-1}{\bar{x}_{2}-1}=B \frac{\bar{x}_{1}^{n}-1}{\bar{x}_{1}-1} .
$$

Применим к (6) эндоморфизм $\psi=\left\{\bar{x}_{1} \mapsto 1, \bar{x}_{2} \mapsto \bar{x}_{2}\right\}$. Тогда

$$
\frac{\bar{x}_{2}^{n l}-1}{\bar{x}_{2}-1}=n \cdot \psi(B) \text {. }
$$

Так как (7) невозможно при $n \geqslant 2$, центр групшы $G_{n}=\left\langle x_{1}, x_{2}: x_{1}^{n}=1, \mathfrak{A}^{2}\right\rangle$ тривиален при $n \geqslant 2$.

2. Пусть $G$ - группа такая, что целочисленное групповое кольцо $\mathbb{Z}(G)$ не имеет делителей нуля, $N$ - нормальная подгруппа из $G$ и $G / N$ - свободная абелева групша ранга 2 с порождающими $x N$ и $y N$.

Элемент $y$, действуя на подгруппу $N$ сопряжением, индуцирует автоморфизм $\alpha$ кольца $\mathbb{Z}(N)$. Ввиду того, что группа $G / N$ без кручения, каждый элемент $а$ группового кольца группы $\langle N, y\rangle$, порожденной подгруппой $N$ и элементом $y$, однозначно записьвается в виде

$$
a=a_{i} y^{i}+\cdots+a_{i+d} y^{i+d},
$$

где $i, d \in \mathbb{Z}, d \geqslant 0, a_{j} \in \mathbb{Z}(N)$. Таким образом, групповое кольцо $\mathbb{Z}(\langle N, y\rangle)$ является кольцом косых многочленов Лорана $\mathbb{Z}(N)[y]$ с автоморфизмом $\alpha(a)=y^{-1} a y, a \in \mathbb{Z}(N)$.

Аналогично, имеем

$$
\mathbb{Z}(\langle N, x\rangle)=\mathbb{Z}(N)[x], \quad \mathbb{Z}(G)=\mathbb{Z}(\langle N, y\rangle)[x]=\mathbb{Z}(\langle N, x\rangle)[y]
$$

Рассмотрим кольцо косых рядов Лорана от переменной $y$ над кольцом $\mathbb{Z}(N)$. Элементы этого кольца - формальные суммы вида $a_{i} y^{i}+a_{i+1} y^{i+1}+\cdots$, где $i \in \mathbb{Z}$, 
$a_{j} \in \mathbb{Z}(N)$. Обозначим это кольцо через $\mathbb{Z}(N)[[y]]$. Заметим, что $\mathbb{Z}(N)[y]$ является подкольцом $\mathbb{Z}(N)[[y]]$.

Так как $G^{\prime} \leqslant N$, сопряжение элементом $x$ определяет автоморфизм кольца $\mathbb{Z}(N)[y]$. Расширяя этот автоморфизм до автоморфизма кольца $\mathbb{Z}(N)[[y]]$, мы получим кольцо косых многочленов $\mathbb{Z}(N)[[y]][x]$, ненулевыми элементами которого являются суммы вида

$$
a=a_{i} x^{i}+\cdots+a_{i+d} x^{i+d},
$$

где $i, d \in \mathbb{Z}, d \geqslant 0, a_{j} \in \mathbb{Z}(N)[[y]], a_{i}, a_{i+d} \neq 0$.

Назовем $d$ cmепенью элемента $a$ по переменной $x$. Таким образом, степень по $x$ определена для трех колец:

$$
\mathbb{Z}(\langle N, x\rangle)=\mathbb{Z}(N)[x], \quad \mathbb{Z}(G)=\mathbb{Z}(\langle N, y\rangle)[x], \quad \mathbb{Z}(N)[[y]][x] .
$$

Однако, эти определения дают один результат для общих элементов колец.

Доказательство следующей леммы можно найти в [7].

ЛЕмма. Предположим, что $a, b \in \mathbb{Z}(G)$ и $a-(1-x)(1-y)^{-1} b$ имеет нулевую

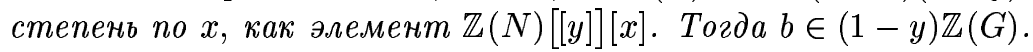

Теперь мы можем доказать следующий результат.

Теорема 2. Пусть $F$ - свободная группа конечного ранга не менее $2, T$ - нормальная подгруппа из $F$, принадлежсащая коммутанту $F^{\prime}$ группь $F, f$ - элемент из $F^{\prime}, R=\langle f\rangle^{F}$ - нормальное замыкание әлемента $f$ в группе $F$. Предположим, что иентр группь $G=F / T R$ тривиален и кольио $\mathbb{Z}(G)$ не содерэмит делителей нуля. Тогда иентр группы $H=F / T^{\prime} R$ также тривиален.

ДоказАтЕльство. Обозначим через $M$ группу $T R$. Тогда $T^{\prime} R=M^{\prime} R$, и нам достаточно доказать тривиальность центра группы $F / M^{\prime} R$ при условии, что центр группы $G=F / M$ тривиален, кольцо $\mathbb{Z}(G)$ не имеет делителей нуля и элемент $f$ взят из подгруппы $M$, принадлежащей коммутанту $F^{\prime}$.

Заметим прежде всего, что если группа $H$ имеет центральньй элемент, то он лежит в подгрупше $M / M^{\prime} R$.

Действительно, пусть взаимньй коммутант $[z, F]$ лежит в $M^{\prime} R$. Применим к включению $[z, F] \leqslant M^{\prime} R$ естественный гомоморфизм групшы $F$ на групш $G$. Обозначим через $\bar{z}$ образ элемента $z$ при этом гомоморфизме. Так как $R \leqslant M$, имеет место $[\bar{z}, G]=1$. Но центр группы $G$ тривиален. Поэтому $z \in M$.

Пусть $x_{1}, \ldots, x_{r}-$ свободные порождающие свободной групшы $F$. Элементы $\left[z^{-1}, x_{i}^{-1}\right]$ лежат в $M^{\prime} R$. Так как $z \in M$, в кольце $\mathbb{Z}(F / M)$ имеем

$$
\left(1-\bar{x}_{i}\right) \partial_{j} z=\partial_{j}\left[z^{-1}, x_{i}^{-1}\right], \quad j, i=1, \ldots, r .
$$

Согласно лемме из [8] элемент $\left[z^{-1}, x_{i}^{-1}\right]$ лежит в подгруппе $M^{\prime} R$ тогда и только тогда, когда

$$
\partial_{j}\left[z^{-1}, x_{i}^{-1}\right]=\bar{\gamma}_{i} \partial_{j} f+\bar{\gamma}_{i j}, \quad j=1, \ldots, r,
$$

где $\bar{\gamma}_{i} \in \mathbb{Z}(F / M), \bar{\gamma}_{i j}$ - элементы идеала $I_{f}$, порожденного элементом $\bar{f}-1$ в кольце $\mathbb{Z}(G)$. Так как $f \in M$, то $\bar{\gamma}_{i j}=0$ при всех $i=1, \ldots, r$. Поэтому из (8) и (9) получаем

$$
\left(1-\bar{x}_{i}\right) \partial_{j} z=\bar{\gamma}_{i} \partial_{j} f, \quad i=1, \ldots, r .
$$


В группе $G$ рассмотрим подгруппу $N$, порожденную элементами $\bar{x}_{3}, \ldots, \bar{x}_{r}$ и коммутантом $G^{\prime}$. Тогда $G / N$ - свободная абелева группа ранга 2 .

Из (10) при $i=1,2$ получаем

$$
\partial_{j} z=\left(1-\bar{x}_{1}\right)^{-1} \bar{\gamma}_{1} \partial_{j} f, \quad \partial_{j} z=\left(1-\bar{x}_{2}\right)^{-1} \bar{\gamma}_{2} \partial_{j} f, \quad j=1, \ldots, r,
$$

где $\left(1-\bar{x}_{1}^{-1}\right)^{-1} \bar{\gamma}_{1} \partial_{j} f$ и $\left(1-\bar{x}_{2}\right)^{-1} \gamma_{2} \partial_{j} f$ - элементы из кольца $\mathbb{Z}(\langle N\rangle)\left[\left[\bar{x}_{1}\right]\right]\left[\left[x_{2}\right]\right]$. Из $(11)$ получаем

$$
\left(1-\bar{x}_{1}\right)^{-1} \bar{\gamma}_{1} \partial_{j} f=\left(1-\bar{x}_{2}\right)^{-1} \bar{\gamma}_{2} \partial_{j} f
$$

Тогда

$$
\left(1-\bar{x}_{2}\right)\left(1-\bar{x}_{1}\right)^{-1} \bar{\gamma}_{1} \partial_{j} f=\bar{\gamma}_{2} \partial_{j} f
$$

Таким образом,

$$
\left(\left(1-\bar{x}_{2}\right)\left(1-\bar{x}_{1}\right)^{-1} \bar{\gamma}_{1}-\bar{\gamma}_{2}\right) \partial_{j} f=0 .
$$

Теорема Ауслендера-Линдона утверждает, что если факторгруппа $F / M$ бесконечна, то центр групшы $F / M^{\prime}$ тривиален. Поэтому при $f=1$ центр групшы $F / M^{\prime} R$ тривиален.

Если $f \neq 1$, то выберем $j$ так, что $\partial_{j} f \neq 0$ в $\mathbb{Z}(G)$. Так как кольцо $\mathbb{Z}(N)\left[\left[\bar{x}_{1}\right]\right]\left[\bar{x}_{2}\right]$ без делителей нуля, то

$$
\left(1-\bar{x}_{2}\right)\left(1-\bar{x}_{1}\right)^{-1} \bar{\gamma}_{1}=\bar{\gamma}_{2}
$$

Согласно лемме равенство (12) справедливо при $\bar{\gamma}_{1}=\left(1-\bar{x}_{1}\right) \bar{\gamma}_{1}^{\prime}, \bar{\gamma}_{1}^{\prime} \in \mathbb{Z}(G)$. Тогда из (11) имеем

$$
\partial_{j} z=\bar{\gamma}_{1}^{\prime} \partial_{j} f
$$

в $\mathbb{Z}(G)$ при $j=1, \ldots, r$. По лемме из [8] равенство (13) для элемента $z \in N$ означает, что $z$-элемент из $M^{\prime} R$. Теорема доказана.

Из теорем 1 и 2 получаем

СлЕдСТВИЕ [4]. Пусть $G=F_{r}\left(\mathfrak{A}^{n}\right)-$ свободная разрешимая әруппа ступени $n \geqslant 3$ и ранга $r \geqslant 2, f$ - әлемент из последнего неединичного члена ряда коммутантов. Тогда иентр группы $G /\langle f\rangle^{G}$ тривиален.

\section{СПИСОК ЦИТИРОВАННОЙ ЛИТЕРАТУРЫ}

[1] Коуровская тетрадь. Нерешенные вопросы теории групп. 13-е изд. Новосибирск, 1995.

[2] Тимошенко Е.И. Центр группы с одним определяющим соотношением в многообразии метабелевых групп // Сиб. матем. ж. 1973. Т. 14. №6. С. 1351-1355.

[3] Каргаполов М. И. Избранные труды. Группы. Новосибирск: Наука, 1991.

[4] Красников А.Ф., Тимошенко Е.И. Два замечания о группах вида $F /[N, N] / /$ Деп. ВИНИТИ № 4567-В85. М.: ВИНИТИ, 1985.

[5] Bachmuth S. Automorphisms of free metabelian groups // Trans. Amer. Math. Soc. 1965. V. 118. P. 93-104.

[6] Мальцев А. И. Об уравнении $z x y x^{-1} y^{-1} z^{-1}=a b a^{-1} b^{-1}$ в свободной группе // Алгебра и логика. 1962. Т. 1. № 5. С. 45-50.

[7] Bachmuth S., Formanek E., Mochizuki H. Y. IA-automorphisms of two-generator torsion-free groups // J. Algebra. 1976. V. 40. № 1. P. 19-30.

[8] Тимошенко Е. И. О примитивных элементах свободных групп многообразий $\mathfrak{A N}_{n} / /$ Матем. заметки. 1997. Т. 61. №6. С. 884-889. 